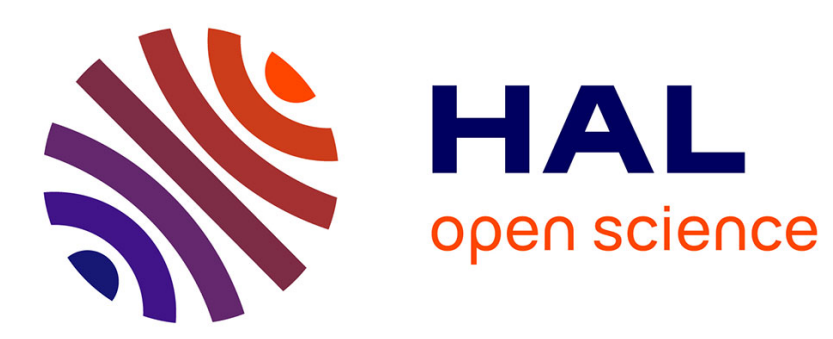

\title{
Possibilité et existentiabilité chez Leibniz
}

Jean-Baptiste Jeangène Vilmer

\section{To cite this version:}

Jean-Baptiste Jeangène Vilmer. Possibilité et existentiabilité chez Leibniz. Revue philosophique de Louvain, 2006, 104 (1), pp.23-45. halshs-00192968

\section{HAL Id: halshs-00192968 \\ https://shs.hal.science/halshs-00192968}

Submitted on 30 Nov 2007

HAL is a multi-disciplinary open access archive for the deposit and dissemination of scientific research documents, whether they are published or not. The documents may come from teaching and research institutions in France or abroad, or from public or private research centers.
L'archive ouverte pluridisciplinaire HAL, est destinée au dépôt et à la diffusion de documents scientifiques de niveau recherche, publiés ou non, émanant des établissements d'enseignement et de recherche français ou étrangers, des laboratoires publics ou privés. 


\title{
Possibilité et existentiabilité chez Leibniz
}

\author{
Jean-Baptiste JEANGENE VILMER \\ EHESS / Université de Montréal \\ $\rightarrow$ publié dans Revue philosophique de Louvain, 104:1, 2006, p. 23-45.

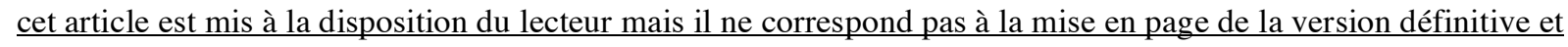 \\ publiée à laquelle il convient de se référer pour toute citation
}

\begin{abstract}
RÉSUMÉ - On sait que l'ontologie leibnizienne trace, pour ainsi dire, le plus court chemin de la possibilité à l'existence. On mesure moins que cette ligne est parcourue d'étapes dont la première, assurément, est l'existentiabilité des possibles. Cet article examine d'abord la conception leibnizienne de la possibilité, à travers cinq définitions. Il analyse ensuite la nature et le rôle de cette existentiabilité, dans ses rapports à la possibilité d'une part et à l'existence elle-même d'autre part, pour finalement conclure sur l'existentialisme d'un auteur dont on souligne habituellement l'essentialisme. De cette manière, possibilité et existentiabilité permettent de redécouvrir à nouveaux frais tout un pan de la pensée de Leibniz.
\end{abstract}

ABSTRACT - It is well-known that Leibniz's ontology as it were traces the shortest path from possibility to existence. The fact is measured less that this line is covered by stages, the first of which is assuredly the existentiability of possibles. This article first examines Leibniz's conception of possibility by means of five definitions. It then analyses the nature and role of this existentiability in its relations to possibility, on the one hand, and existence, on the other, and concludes finally with the existentialism of an author whose essentialism is usually emphasized. In this way possibility and existentiability make it possible to discover again a whole area of Leibniz's thought (translated by J. Dudley). 
La métaphysique leibnizienne est un récit, une histoire : celle de la réalité. L'auteur, pour nous la conter, emprunte un escalier. Sur l'horizon du nihil, il interpelle l'aliquid: pourquoi y a-t-il quelque chose plutôt que rien? Puis, sur la marche de l'aliquid, il somme l'existens : pourquoi existe-t-il telle chose, plutôt que telle autre $?^{1}$ La métaphysique leibnizienne est un itinéraire, un parcours: celui de la possibilité vers l'existence. Les égarements de l'écriture et l'inévitable dilution à laquelle est soumise toute œuvre n'ont pas permis à Leibniz de présenter une théorie unifiée de la possibilité et de l'existence, deux notions qui constituent respectivement le départ et l'arrivée de son ontologie. Les définitions sont éparpillées, les relations esquissées seulement. Il n'est pas d'édifice plus fragile et plus important à la fois.

Nous examinerons ici les deux premières étapes du chemin de la possibilité vers l'existence. Quelle est la conception leibnizienne de la possibilité ? Comment peut-on la définir ? Après avoir étudié la possibilité en tant que telle, nous verrons dans quelle mesure l'existence en fait déjà partie : cette possibilité est déjà possibilité d'exister, tous les possibles tendent à l'existence. C'est ce que l'on peut nommer l'existentiabilité. Possibilité et existentiabilité posent ainsi les premières pierres d'une ontologie leibnizienne qui peut être définie comme le plus court chemin de la possibilité à l'existence ${ }^{2}$.

\section{I- LA POSSIBILITE}

Pourquoi commencer par la possibilité ? Tout simplement parce qu'il s'agit, de l'aveu même de Leibniz, d'une notion primitive : «Ainsi de toutes les choses qui sont actuellement, la possibilité même ou impossibilité d'être est la première $»^{3}$. Ce qui, chronologiquement, se rencontre d'abord n'est certes pas la possibilité (possibilitas) mais les possibles (possibilia); aussi faudra-t-il abstraire les définitions de la possibilité du comportement des possibles. En ce sens nous pouvons dire avec S. Madouas que «Ce que Leibniz appelle la Possibilité (Possibilitas) c'est la possibilité logique, c'est la vérité des possibilia $»^{4}$. Puisant sa raison d'être dans le divers, la possibilité leibnizienne, si elle est une, se décline en plusieurs modes, selon différents attributs : le concept est particulièrement polysémique. Leibniz lui-même rappelle à Spinoza que «les choses sont possibles en beaucoup de manières $»^{5}$. De ces manières nous déduirons cinq définitions. La plupart des confusions naissent de ce que l'on veut réduire la possibilité à l'une de ces définitions, quand au contraire ces définitions ne sont que des expressions, dans des modes différents, via des attributs différents, de la même substance. Ainsi n'est-il pas faux de dire, par exemple, que le critère de la possibilité est un critère $\operatorname{logique}^{6}$, mais il serait faux de $n$ 'en faire $q u^{\prime}$ 'un critère logique.

\footnotetext{
1 Il s'agit de répondre aux deux questions fondamentales posées dans les Principes de la Nature et de la Grâce: «pourquoi il y a plutôt quelque chose que rien? (...) De plus, supposé que des choses doivent exister, il faut qu'on puisse rendre raison, pourquoi elles doivent exister ainsi, et non autrement » (Principes de la Nature et de la Grâce, §7, in Leibniz, 1996, p. 228).

${ }^{2}$ La dernière étape, l'existence elle-même, fait l'objet d'un article distinct, intitulé «L'existence leibnizienne », à paraître dans les Archives de philosophie.

${ }^{3}$ Lettre à Foucher de 1676 ; GP I 370, 12. GP est l'abréviation usuelle des œuvres philosophiques, et GM celle des œuvres mathématiques, dans l'édition Gehardt. Voir la bibliographie pour la référence exacte.

${ }^{4}$ S. Madouas, 1999, p. 364.

${ }^{5}$ Leibniz, 1999, p. 31.

${ }^{6}$ F. B. D'Agostino, 1994, p. 246 : « The criterion of possibility is, then, a logical one : all substances are possible whose complete individual concepts do not involve logical self-contradiction ».
} 
JB Jeangène Vilmer, « Possibilité et existentiabilité... », Revue philosophique de Louvain, 2006

\section{Définition 1 (critère logique) : la possibilité est la non contradiction}

Leibniz établit l'équivalence de la possibilité et de la non contradiction de deux manières : par la via positiva (la possibilité est ce qui n'implique pas contradiction) et par la via negativa (l'impossibilité est ce qui implique contradiction). Dans quel ordre les exposer? La priorité chronologique de la via positiva n'est pas suggérée que par le seul bon sens : c'est, dans les faits, la manière pratiquée par l'auteur, qui souvent fait suivre la définition de la possibilité de celle de l'impossibilité 7 . C'est également, et surtout, un point de philosophie, car la racine de ce choix réside dans la présomption de possibilité : " toujours il y a présomption du côté de la possibilité : c'est-àdire toute chose est tenue possible jusqu'à ce qu'on en prouve l'impossibilité »; « la possibilité est toujours présumée et doit être tenue pour véritable jusqu'à ce qu'on en prouve l'impossibilité ${ }^{8}$. Commençons donc par la via positiva, l'équivalence positive de la possibilité et de la non contradiction.

Les occurrences d'une telle équivalence peuvent se regrouper en trois formulations génériques: "Possibilis quae non est impossibilis ${ }^{9}$, "Possibilia sunt, quae non implicant contradictionem ${ }^{10}$, et "Possibile est quod non continet contradictiorum ${ }^{11}$. La relation d'équivalence est alors signifiée par trois verbes différents : « est», «implicant» ou « continet». La possibilité est, implique ou contient la non contradiction.

Le terme d'implication doit nous alarmer sur un point. L'équivalence est logiquement définie par la double implication : $(\mathrm{A} \equiv \mathrm{B}) \leftrightarrow \forall \mathrm{x},(\mathrm{x} \in \mathrm{A} \rightarrow \mathrm{x} \in \mathrm{B}) \wedge(\mathrm{x} \in \mathrm{B} \rightarrow \mathrm{x} \in \mathrm{A})^{12}$. Si donc l'on veut montrer que l'implication dont il est question (la possibilité implique la non contradiction) désigne une équivalence, il faut pouvoir montrer l'implication réciproque : la non contradiction implique la possibilité, ou tout ce qui n'est pas contradictoire est possible. Le peut-on ? Oui, Leibniz use également de l'implication réciproque : «Je demeure d'accord du principe de M. Bayle, et c'est aussi le mien, que tout ce qui n'implique point de contradiction est possible $\gg^{13}$. Aussi pouvonsnous conclure que les différentes formulations utilisées par l'auteur (la possibilité est, implique ou contient la non contradiction) sont équivalentes entre elles, ce qui d'ailleurs conforte l'interprétation selon laquelle l'implication leibnizienne doit être interprétée en termes d'inclusion, plus précisément d'inclusion du prédicat dans le sujet ${ }^{14}$. De cette manière la possibilité est ce qui n'implique pas contradiction, et c'est la raison pour laquelle l'auteur peut définir les «mondes possibles » comme étant des « séries de $\operatorname{choses}^{15}$ qui n'impliquent pas contradiction » : «Autant de

\footnotetext{
${ }^{7}$ Par exemple en Théodicée, II, §174, en reprenant Bayle.

8 Lettre à la princesse Elisabeth (?) de fin 1678, in Leibniz, 1940, p. 58 ; et Lettre à Jacquelot du 20 novembre 1702 (Raisons que M. Jacquelot m'a envoyées pour justifier l'Argument contesté de des-Cartes qui doit prouver l'existence de Dieu, avec mes réponses), GP III 444.

${ }^{9}$ Leibniz, 1903, p. 387, souligné par nous (spn). Voir aussi Generales Inquisitiones, 2.

10 GM III 574, spn. Formulations similaires en Generales Inquisitiones, 2 ; en A VI-1 514 et 540 et A VI-2 495 ; Lettre à Bernoulli du 21 février 1699, GM III 574. A est l'abréviation usuelle pour les Sämtliche Schriften und Briefe de l'Akademie. Voir la bibliographie pour la référence exacte.

${ }^{11}$ Generales Inquisitiones, 2, in Leibniz, 1903, p. 364, spn.

12 A est équivalent à $\mathrm{B}$ si et seulement si, quelque soit $\mathrm{x}$, si x appartient à $\mathrm{A}$, alors $\mathrm{x}$ appartient à $\mathrm{B}$, et si x appartient à $\mathrm{B}$, alors x appartient à $\mathrm{A}$.

13 GP VI 252.

14 Comme le note G. Roncaglia, 1988, p. 45, après avoir remarqué que Leibniz préfère même utiliser «continet » à «implicat».

15 L'expression «systèmes de choses » in Leibniz, 1948, p. 315. Notre but ici n'est pas de pénétrer le débat qui s'est très largement construit dans la littérature anglo-saxonne autour de la notion de «monde possible » et qui a conduit à l'élaboration de véritables «Possible Worlds Semantics ». Comme le montre G. Kalinowski, 1983, ces sémantiques qui se réclament de la notion leibnizienne de «monde possible » surinterprètent Leibniz plus qu'elles n'en suivent le texte.
} 
séries de choses n'impliquant pas de contradiction peut-on fabriquer (fingere), autant y a-t-il de mondes possibles $\gg 16$.

Notons sans y consacrer de développement qu'il en va de même pour la via negativa, c'est-àdire l'équivalence négative de l'impossibilité et de la contradiction, pour laquelle l'on retrouve les formulations habituelles : «Impossibile est quod involvit contradictionem », «Si A explicando prodit B non B, A est impossibile», ou encore "Quod continet B non B, idem est quod impossibile $»^{17}$.

Après avoir cité ce que Bayle présente comme une maxime («Tout ce qui implique contradiction est impossible, et tout ce qui n'implique point contradiction est possible »), Leibniz ajoute : « ce qu'on vient de marquer comme une maxime est même la définition du possible et de $1^{\prime}$ impossible $\gg^{18}$. De cette définition du possible, il va déduire, à la fin de la Théodicée (\$413), le concept des mondes possibles, à travers le mythe de Sextus.

\section{Définition 2 (critère métaphysique) : la possibilité est l'essence}

Equivalence fondamentale, que l'auteur établit généralement par la définition de l'essence. L'essence est la possibilité : «L'essence dans le fond n'est autre chose que la possibilité de ce qu'on propose $»^{19}$. L'ens est le possibile: «Etre, c'est-à-dire possible ${ }^{20}$; « $\hat{\mathrm{ETRE}}$ : terme possible $»^{21}$. Nombreuses, effectivement, sont les occurrences où l'on trouve soit l'essence définie en terme de possibilité, soit «essence » et «possibilité » simplement utilisés comme synonymes (par exemple : « les essences ou possibilités » en Monadologie, §44) ${ }^{22}$. Dire de la possibilité qu'elle est l'essence, c'est du même coup dire qu'elle est la positivité, car l'auteur établit une seconde équivalence entre être et positif : «Le positif est identique à l'ÊTRE $»^{23}$.

On a donc la ligne suivante : possible $\equiv$ être $\equiv$ positif. Et l'on pourrait y joindre la réalité de la manière suivante : le réel est tout ce qui est positif, «Realitas est praedicatum positivum ${ }^{24}$, c'està-dire tout ce qui est, donc tout ce qui est possible.

\section{Définition 3 (critère épistémologique) : la possibilité est l'intelligibilité distincte}

Il n'est pas, dans les textes, question de «l'intelligibilité distincte », mais du " pensable distinctement ». Le choix, qui est le nôtre, du substantif " intelligibilité » plutôt que " pensabilité » est motivé par des raisons purement esthétiques, et ne dénote en rien une différence entre les deux termes. C'est donc bien de la démonstration de ce que la possibilité est l'intelligibilité

\footnotetext{
16 Conversation avec Wagner, mars 1698, in Leibniz, 1948, p. 389.

17 Respectivement Leibniz, 1903, p. 253 ; Leibniz, 1970, p. 56 et Leibniz, 1903, p. 368. Voir également : « Je dis nécessairement, c'est-à-dire en sorte que le contraire implique contradiction, ce qui est le véritable et unique caractère de l'impossibilité. En outre, de même qu'à l'impossible répond le nécessaire, à la proposition impliquant contradiction répond la proposition identique, car dans les propositions, l'impossible premier est : A n'est pas A, de même que le premier nécessaire est : A est $A$ » (Lettre à Conring du 19 mars 1678, in Leibniz, 2001a, pp. 138-139).

${ }^{18}$ Théodicée, II, $\$ 174$ (la citation de Bayle clôt le $\$ 173$ ).

${ }^{19}$ Nouveaux Essais, III, III, 15 ; GP V 272, orthographe corrigée par nous (ocpn).

${ }^{20}$ Recherches générales sur l'analyse des notions et des vérités, in Leibniz, 1998, p. 211.

${ }^{21}$ Définitions (1679-1686), in Leibniz, 1998, p. 108.

22 Voir Lettre à Simon Foucher, GP I 370 ; Definitiones, in Leibniz, 1948, I, p. 324 ; De iis, quae per se concipiuntur, GP I 271 ; Leibniz, 1903, pp. 259, 360, 259, 271, 376, 392 ; Leibniz, 1948, pp. 325, 326 ; Jagodinski, 1913 , p. 8.

${ }^{23}$ Leibniz, 1998, p. 201. L'être est littéralement ce dont le concept enveloppe quelque chose de positif : «Ens est, cujus conceptus aliquid positivi involvit sive quod a nobis concipi potest, modo id quod concipimus sit possibile nec involvat contradictionem (...) »(GP VII 319). Voir aussi : «Ens, seu pure positivum (Leibniz, 1948, p. 325); «Positivum idem est quod Ens » (Leibniz, 1903, p. 356).

24 Note sur une lettre d'Eckhard, GP I 226.
} 
JB Jeangène Vilmer, « Possibilité et existentiabilité... », Revue philosophique de Louvain, 2006

distincte qu'il s'agit en montrant que le possible est le pensable de manière distincte. Pour ce faire, nous procéderons en deux temps, en découpant l'expression " pensable de manière distincte » en son genre (le pensable) et sa différence spécifique (de manière distincte).

1. Le possible n'est pas seulement le pensable. Ce qui est possible est certes pensable: «Possibile est quicquid potest intelligi » 25 . L'ensemble «possible est inclu dans l'ensemble «pensable », c'est-à-dire que le genre du possible est le pensable. Mais cela suffit-il pour le caractériser ? Oui, si le possible n'est que le pensable, si donc il épuise son genre, en se confondant avec lui. Non, s'il ne s'y réduit pas. Pour le savoir, examinons la question suivante : Tout ce qui est pensable est-il possible ? La réponse de Leibniz est clairement négative : "non omnia, de quibus cogitamus, esse possibilia $»^{26}$, et la raison en est que « Oui sans doute on pense quelques fois à des choses impossibles $\gg^{27}$ - à des choses impossibles, à travers les expressions classiques de l'infini par exemple (la vitesse la plus grande, le nombre de tous les nombres), et non à l'impossible luimême : «Cependant, au premier abord, il peut sembler que nous possédons l'idée du mouvement le plus rapide ; car nous comprenons parfaitement ce que nous disons, et néanmoins de choses impossibles nous ne possédons absolument aucune idée $»^{28}$.

Par cette étape que tout ce qui est pensable n'est pas possible, Leibniz écarte la réduction de la possibilité à la simple pensabilité. Putnam fait de même lorsqu'il écrit : " it is conceivable that water isn't $\mathrm{H}_{2} \mathrm{O}$. It is conceivable but it isn't possible ! Conceivability is no proof of possibility $\gg^{29}$. Ainsi le possible n'est-il pas seulement le pensable : il faut compléter le genre par une différence spécifique.

2. Le possible est le pensable de manière distincte. La différence spécifique en question est que le pensable, pour être le possible, doit être un pensable de manière distincte. Il y a deux façons de s'en apercevoir. La première est directe. Leibniz utilise différents adjectifs qualificatifs, notamment «parfaitement» ou «distinctement », pour la manifester : «J'appelle possible tout ce qui est parfaitement concevable ${ }^{30}$; "Possibile est quicquid clare distincteque cogitabile est*. Impossible contra $»^{31}$; «que ces Mondes soient possibles ou, ce qui est la même chose, intelligibles distinctement $»^{32} ;$ 《distincte cogitabile est, sive possibilis $»^{33}$. Et cette clarté et distinction implique vérité et certitude, car «Verum est quicquid clare distincteque est. ((Certum) est quicquid clare distincteque sentitur. Seu certitudo est claritas veritatis) $»^{34}$.

\footnotetext{
25 Et «Impossibile est quod intelligi non potest, sive quod contradictionem involvit, ut Triangulum quadrilaterum » (Definitiones : aliquid, nihil, opposita, possibile, août 1688 - janvier 1689 ?, A VI-4-A 937).

${ }^{26}$ Colloquium cum Dno. Eccardo Professore Rintelensi Cartesiano, praesente Dni. Abbatis Molani fratre, 5 avril 1677, GP I 213.

${ }^{27}$ Lettre à la princesse Sophie, GP IV 293, ocpn. Voir Lettres à Malebranche, 22 juin 1679, GP I 331 sq. ; Meditationes de Cognitione, Veritate et Ideis, GP IV 424 ; Lettre à Heinrich Oldenburg, 16/26 avril 1673, GP VII 9, 28 décembre 1675, GM I 85 ; De Synthesi et Analysi universali seu Arte inveniendi et judicandi, GP VII 294 ; Lettre à Bernouilli, GM III 535 ; Jagodinski, 1913, p. 8.

${ }^{28}$ Meditationes de Cognitione, Veritate et Ideis, GP IV 424, in Leibniz, 2001b, pp. 21-23.

${ }^{29}$ H. Putnam, 1973, p. 709. Par opposition à Wittgenstein qui, dans le Tractatus, 3.02, écrit : «La pensée contient la possibilité de l'état de choses qu'elle pense. Ce qui est pensable est également possible » (1961, p. 36).

${ }^{30}$ Lettre à Bourguet de décembre 1714, GP III 573-574, spn.

${ }^{31}$ Vorarbeiten zur Characteristica Universalis. Definitionentafel, 1671-1672, A VI-2 494, spn. * en note est rappelée la définition 1 : «Possibile est quod non implicat contradictionem » (n. 46).

32 GP V 246, spn.

33 Leibniz, 1903, p. 77, spn. Voir également Lettre à Louis Bourguet, décembre 1714, GP III 573 ; Meditationes de Cognitione, Veritate et Ideis, GP IV 424 ; Discours de métaphysique, XXIV sq., GP IV 449 sq. ; Essais de Théodicée, 26, GP VI 432.

${ }^{34}$ Vorarbeiten zur Characteristica Universalis. Definitionentafel, 1671-1672, A VI-2 493.
} 
La seconde façon de mettre en évidence cette différence spécifique est indirecte, elle consiste à transiter par la définition de l'être, dans le syllogisme suivant : on sait que le possible est l'être (définition 2), or «L'ÊTRE est le pensable de manière distincte ${ }^{35}$, donc le possible est le pensable de manière distincte. Quelle est cette manière distincte ? Là encore, transiter par l'être s'avère utile. L'être (comme le possible) est ce qui peut être conçu : "L'être est ce qui peut être conçu à propos de a, b, c, quels qu'ils soient ». Mais plus précisément, ce qui peut être conçu sans contradiction : «L'être est ce qui est conçu consentienter, c'est-à-dire en tant qu'il n'implique pas contradiction $»^{36}$. Ainsi se recoupent les trois définitions de la possibilité : le possible est l'être (définition 2), ce qui peut être conçu distinctement (définition 3), c'est-à-dire ce qui n'implique pas contradiction (définition 1). «Possibile est quicquid distincte cogitari potest, seu cujus notio non involvit contradictionem $»^{37}$.

Nous pouvons donc conclure, avec H. H. Knecht, que « ce qui caractérise le possible, ce n'est pas le fait de pouvoir être pensé, (...) mais de pouvoir être conçu de manière adéquate ${ }^{38}$.

\section{Définition 4 (critère physique) : la possibilité est-elle la probabilité ?}

Concernant la question de savoir si la possibilité peut être définie comme probabilité, il est un débat fameux qui a opposé, en 1971, I. Hacking et M. Wilson. L'un comme l'autre, cependant, ont assimilé la faisabilité (makeability) ou facilité et la probabilité (probability, doctrine of chances) : pris comme synonymes chez Wilson ( probability or makeability »39), ils ne sont distingués chez Hacking que pour montrer comment «What is facile in re corresponds to what is probable in mente ${ }^{40}$. Or, il nous semble préférable, au moins méthodologiquement, de les considérer distinctement, car le résultat ne sera pas le même pour l'un et l'autre : la faisabilité est indiscutable, la probabilité l'est beaucoup moins.

La faisabilité, à condition qu'elle soit entendue comme facilité, peut sans aucun doute être considérée comme une définition leibnizienne de la possibilité, tout simplement parce que quelque chose de possible arrive plus facilement qu'une chose impossible : "Quia facilius evenit aliquid possibile quam impossibile esse $\gg^{41}$. Ainsi l'auteur peut-il prendre facilité et possibilité comme synonymes : "Si les éventualités sont d'égale facilité, c'est-à-dire d'égale possibilité » ${ }^{42}$. Et M. Parmentier, qui explique qu' « est possible ce qui comporte moins de réquisits que son contraire », note : "Cette équation entre le possible et le faisable ou le réalisable (facilis) montre bien que la définition leibnizienne du probable n'a que peu de rapport avec le possible métaphysique. En remplaçant éventualité par condition, cette formule pourrait énoncer un principe du droit constitutionnel $»^{43}$.

La probabilité, quant à elle, fait davantage problème. Certains interprètes, et en tout premier lieu I. Hacking, définissent purement et simplement la possibilité leibnizienne en terme de probabilité, et en font la preuve d'un lien étroit entre ontologie et physique : «I am glad to find that

\footnotetext{
35 Définitions (1679-1686), in Leibniz, 1998, p. 110.

${ }^{36}$ Leibniz, 1998, p. 189.

${ }^{37}$ Definitiones : aliquid, nihil, possibile, positivum, août 1688 - janvier 1689 ?, A VI-4-A 938.

${ }^{38}$ H. H. Knecht, 1981, p. 228.

${ }^{39}$ M. Wilson, 1971, pp. 615-616.

40 I. Hacking, 1971, p. 603, qui s'appuie sur «Facile est valde possibile, seu cujus pauca sunt requisita. Quod facile est in re, id probabile est in mente $»$ (A VI-2 492).

${ }^{41}$ Elementa Juris Naturalis, 1671 (?), A VI-1 471.

42 De incerti Aestimatione, sept. 1678, in Leibniz, 1995, p. 164.

43 Leibniz, 1995, respectivement p. 11 et p. 164, n. 88.
} 
Mahnke ${ }^{44}$ anticipated my interpretation, and took the probability-possibility-facility-creatibility nexus as a final proof of the way that Leibniz linked ontology and physics ${ }^{45}$. Ce qui pose problème n'est pas tant le lien, indiscutable, que l'unité interne du concept de possibilitéprobabilité. Hacking s'appuie sur une seule expression tirée du De incerti aestimatione de Leibniz, qui définit la probabilité en terme de possibilité : «Probabilitas est gradus possibilitatis ${ }^{46}$. Aussi la probabilité implique-t-elle effectivement la possibilité, et Hacking a raison de montrer comment Leibniz, de cette manière, est à l'origine de la tradition qui définira la probabilité en termes d'équipossibilité 47 . Mais pour obtenir une équivalence, qui nous permettrait de parler d'un concept de «possibilité-probabilité » ayant une unité interne, une seule implication ne suffit pas : comme nous l'avons vu tout à l'heure, l'implication réciproque est nécessaire. Il faudrait donc montrer que non seulement la probabilité implique la (est définie en terme de) possibilité, mais encore que la possibilité à son tour implique la (est définie en terme de) probabilité, et cette seconde proposition est loin d'être établie. Il est même un argument que Wilson objecte à Hacking et qui montre de manière frappante comment cette implication réciproque ne peut être satisfaite : le combat de tous les possibles pour l'existence n'impliquant aucun degré de «chance» ou d'indéterminisme (puisqu'il est réglé par Dieu), il ne saurait en aucune manière y être question de probabilité ${ }^{48}$. Aussi suivons-nous la conclusion de Wilson, contre Hacking : «I do not wish to deny that there may have been some connection in Leibniz's mind between the two realms of discourse. I do however doubt that the metaphysical "propensities » of Leibniz's possibles can be either an underpinning or a reflection of weights assigned to various alternatives in probable reasoning ${ }^{49}$. Tout en reconnaissant l'existence d'un lien étroit entre la possibilité métaphysique et la probabilité (la probabilité implique la possibilité), en l'absence d'implication réciproque donc d'équivalence nous refusons l'unité interne que Hacking semble suggérer.

\section{II- L'EXISTENTIABILITE}

\section{Définition 5 : la possibilité est possibilité d'exister}

Le mot même de " possible », lorsqu'il est à la suite de l'auxiliaire « être », semble appeler déjà en lui l'existence ${ }^{50}$, car être possible, dira-t-on, n'est autre qu'avoir la possibilité d'exister. A rigoureusement parler, cependant, être possible est (n') avoir la possibilité (que) d'être. C'est donc un point de doctrine, et non une évidence grammaticale, si, chez Leibniz, être possible, ce n'est pas avoir la possibilité d'être, mais bien d'exister. Et l'on en peut rendre raison en deux étapes.

D'une part, il ne peut en être autrement dans un système qui identifie la possibilité à l'essence, c'est-à-dire dans lequel être possible est la même chose qu'être (définition 2). Car l'expression « être possible » est alors redondante en ce qu'elle ne signifie rien d'autre qu' " être » : la possibilité ne s'ajoute pas à l'être, elle l'est. De la même manière, l'expression «avoir la possibilité d'être »n'a absolument aucun sens, puisqu'elle présuppose un état dans lequel ce qui a

\footnotetext{
44 I. Hacking, 1971, p. 604, n. 4, renvoie à « Dietrich Mahnke, « Leibnizens Synthese von Universalmathematik und Individualmetaphysik », Jahrbuch für Philosophie une phänomenologische Forschung, VII (1925) : 305-611, esp. P. 384. »

${ }^{45}$ I. Hacking, 1971, pp. 602-604.

${ }^{46}$ Notons qu'on retrouve la même formule chez B. Van Fraassen, 1977, p. 159 : «What is probable is a gradation of the possible».

${ }^{47}$ I. Hacking, 1975.

${ }^{48}$ M. Wilson, 1971, p. 612.

${ }^{49}$ M. Wilson, 1971, pp. 615-616. C'est la position également adoptée par L. Krüger, 1981, pp. 58-59.

50 M. Guéroult, 1947 , p. 63 : «le possible, dont le nom même implique déjà une référence, si ténue qu'elle soit, à l'existence ».
} 
la possibilité d'être n'est pas encore, état pré-ontologique exclu par l'ontologie leibnizienne dont le donné, le premier niveau, qui donc n'en connaît pas d'antérieur, est celui de l'être possible, en tant qu'être-possible, c'est-à-dire en tant que l'être et le possible ne font qu'un, ne sont qu'une seule et même chose. Ainsi « être possible », chez Leibniz, ne peut-il se traduire par "avoir la possibilité d'être », comme le voudrait pourtant la grammaire.

D'autre part, donc, et plutôt que de se rendre à l'évidence grammaticale qui traduit directement «être possible» en "avoir la possibilité d'être", il faut pour comprendre Leibniz passer par une étape intermédiaire : être possible, c'est avoir la possibilité de. Dire qu'un possible est possible, est donc dire qu'il a la possibilité de. Or, de quoi, précisément, a-t-il la possibilité ? D'exister. Dans la doctrine leibnizienne, le possible est possible en ce qu'il a la possibilité d'exister. C'est ainsi qu'être possible, pour Leibniz, n'est pas autre chose qu'avoir la possibilité d'exister.

Nous suivons sur ce point l'excellente analyse que fait $M$. Heidegger dans son bref commentaire des 24 Thèses métaphysiques ${ }^{51}$ :

La possibilité d'un possible est, en tant qu'être, déjà un " exister », c'est-à-dire se rapportant par essence à l'existentia. Le possible est déjà, parce qu'il n' " est » qu'en tant que tel absolument ce qu'il est, quelque chose se pouvant vouloir [Mögendes], une propension à s'essayer soi-même [vorgeneigtes Sichversuchen] et ainsi un fonder et un effectuer. L'être-possible conçu et concevable seulement à partir de l'essence de l'Être provoque en soi l'appétition re-présentante et cela de telle sorte que ce provoquer est déjà un pro-duire au-dehors [Herausführen] et un exécuter (ex-sequi), effectuer [Ausführen] de l'existentia ${ }^{52}$.

\section{L'analyticité de l'existence (l'internalité)}

C'est en ce sens, parce qu'être possible n'est autre qu'avoir la possibilité d'exister, que s'affirme la tendance ou la prétention du possible vers l'existence ${ }^{53}$. C'est effectivement en vertu de ce qu'ils contiennent déjà en eux, mus par une force interne, que tous les possibles tendent à l'existence, qu' « il y a, dans les choses possibles ou dans la possibilité même, c'est-à-dire dans l'essence, une certaine exigence d'existence, ou bien, pour ainsi dire, une prétention à l'existence, en un mot, que l'essence tend par elle-même à l'existence ${ }^{54}$. Si c'est par elle-même que l'essence tend à l'existence, c'est que l'existence est déjà contenue ${ }^{55}$ dans la définition même de l'essence

\footnotetext{
51 M. Heidegger, 1971, pp. 350-365.

52 M. Heidegger, 1971, pp. 357-358.

53 Vers l'existence et non vers l'être, bien entendu. Il semble donc n'y avoir aucun sens, d'un point de vue leibnizien, à parler, comme le fait J.-P. Sartre, 1943, II, I, 4, p. 136, de « donner une tendance vers l'être aux possibles » (spn). C'est qu'à cet instant l'auteur ne parle sans doute plus au nom de Leibniz mais, sans prévenir, en son sien propre. Si le possible leibnizien tend vers l'existence et non vers l'être, c'est parce que Leibniz ne distingue pas le possible de l'être. Mais il ne faut pas pour autant en déduire que, si le possible sartrien peut tendre, lui, vers l'être et non vers l'existence, c'est parce que Sartre distinguerait, lui, le possible de l'être, car la position sartrienne est sur ce point bien plus proche de Leibniz qu'elle n'en a l'air, comme en témoigne cette explication qui, sans identifier franchement comme le fait Leibniz le possible et l'être, nie la réalité du possible à précéder l'être, et affirme du même coup leur coexistence, sinon chronologique («l'état possible n'est pas encore»), au moins ontologique : « Ainsi, le possible ne saurait se réduire à une réalité subjective. Il n'est pas non plus antérieur au réel ou au vrai. Mais il est une propriété concrète de réalités déjà existantes. Pour que la pluie soit possible, il faut qu'il y ait des nuages au ciel. Supprimer l'être pour établir le possible dans sa pureté est une tentative absurde; la procession souvent citée qui va du non-être à l'être en passant par le possible ne correspond pas au réel. Certes, l'état possible n'est pas encore; mais c'est l'état possible d'un certain existant qui soutient par son être la possibilité et le non-être de son état futur » (ibid., p. 137).

${ }^{54}$ De Rerum originatione radicali, GP VII 303, in Leibniz, 2001b, p. 173, spn.

55 L. Couturat, p. 14 : «En un mot, on peut dire que son existence est inscrite d'avance dans son essence, qu'elle fait partie de sa compréhension ».
} 
JB Jeangène Vilmer, « Possibilité et existentiabilité... », Revue philosophique de Louvain, 2006

comme possibilité, qui est possibilité d'exister. On peut donc parler de l'analyticité de l'existence au sens kantien d'inclusion du prédicat (l'existence) dans le sujet (l'essence) ${ }^{56}$.

\section{Vis, praetensio, exigentia et conatus ad existentiam (la nature de cette internalité)}

Cette force interne se manifeste dans différents mots: Leibniz parle de tendance (vis), de prétention (praetensio) et d'exigence (exigentia) d'exister (ad existentiam). La "prétention» pourrait tout aussi bien s'écrire " pré-tension » car, d'une part, elle n'a de raison d'être qu'en tant qu'elle est un pré-alable (à l'existence en acte) et, d'autre part, cet état par définition provisoire est effectivement un tension, tension de l'être vers l'existence : la praetensio ad existentiam du possible est une pré-tension.

La question qui se pose d'emblée, pour le lecteur spinoziste, est de savoir si cet effort de l'être pour persévérer non dans l'être mais dans ou vers l'existence peut être nommé conatus. La réponse doit être affirmative, sous réserve d'une condition. On trouve effectivement que Leibniz lui-même utilise l'expression «conatus ad existentiam» pour désigner cette tendance, dans la cinquième de ses 24 thèses métaphysiques - ce qui n'a pas échappé à M. Heidegger qui en conclut que «La vis a le caractère du conatus, de l'entreprise tendant déjà à tenter une possibilité » ${ }^{57}$. La réserve réside en ce qu'un tel conatus ne doit pas être entendu en un sens strictement spinoziste, car Leibniz s'oppose tout aussi expressément à Spinoza sur ce point : «C'est à tort que l'auteur appelle la volonté l'effort de chaque chose pour persister dans son être ; car la volonté a des fins plus particulières et tend à un mode plus parfait d'existence. I1 a tort aussi de dire que l'effort est identique à l'essence, tandis que l'essence est toujours la même et que les efforts varient. Je ne saurais admettre que l'affirmation soit l'effort de la raison pour persévérer dans son être, c'est-àdire pour conserver ses idées. Nous avons cet effort même sans rien affirmer $»^{58}$. Le conatus ad existentiam, s'il est, est donc proprement leibnizien. On peut dire de l'essence qu'elle est un conatus enveloppé, et de l'existence qu'elle est un conatus développé : l'être, chez Leibniz, n'est pas immobile, il est dynamique.

L'existentiabilité. Le possible existe déjà, d'une certaine manière. Cette manière d'exister du possible, qui n'est pas encore l'existence, est l'existentiabilité.

Cette prétention vers l'existence est si forte qu'elle signifie que le possible existe déjà, d'une certaine manière, comme le présuppose Leibniz dans cet extrait : «A quoi je réponds que ni les essences ni ce que l'on appelle les vérités éternelles, qui s'y rapportent, ne sont fictives, mais qu'elles existent, pour ainsi dire, dans une région des idées, à savoir en Dieu lui-même qui est la source de toute essence et de l'existence de tous les autres êtres ${ }^{59}$. Les essences « existent (...) pour ainsi dire ». Or, l'on peut substituer « possibles » à « essences » puisque le possible et l'être ne font qu'un. Donc l'auteur affirme ici que les possibles « existent (...) pour ainsi dire ». Voilà qui, à première vue, semble contradictoire si l'on tient, et c'est le cas, à la distinction irréductible entre être et exister, entre possibilité et existence. Mais la difficulté s'efface, la contradiction se réduit en simple paradoxe, si l'on comprend que l'existence-pour-ainsi-dire de tous les possibles dont il est ici question n'est pas l'existence à laquelle accèdent seulement certains d'entre eux. On distinguera donc désormais l'existentiable de l'existant.

\footnotetext{
56 Sans dire pour autant que l'existence est un prédicat ou, du moins, un véritable prédicat, comme nous l'avons montré ailleurs (voir notre article «L'existence leibnizienne », à paraître dans les Archives de philosophie).

${ }^{57}$ M. Heidegger, 1971, t. II, p. 356. Voir également J. Moreau, 1956, p. 77.

58 Réfutation inédite de Spinoza, in Leibniz, 1999, p. 35.

${ }^{59}$ De Rerum originatione radicali, GP VII 305, in Leibniz, 2001b, p. 179.
} 
L'existentiabilité est l'existence-pour-ainsi-dire des possibles, elle est la manière pour le possible d'exister par sa simple possibilité : «Car l'essence de la chose n'étant que ce qui fait sa possibilité en particulier, il est bien manifeste qu'exister par son essence, est exister par sa possibilité ${ }^{60}$. L'existentiabilité est, en somme, et pour reprendre les mots de Heidegger, «le caractère d'existence de la possibilité ${ }^{61}$. Tous les possibles sont existentiables, tous existent d'une certaine manière. La question de la nature de cette manière (comment existent-ils ?) revient à celle du lieu : où existent-ils ? Leibniz, en effet, ne reconnaît l'existentiabilité des possibles qu'en tant qu'elle est localisée : «dans une région des idées, à savoir en Dieu lui-même » ${ }^{62}$. C'est parce que l'entendement divin est la région des essences et des vérités éternelles ${ }^{63}$, qu'il est également le lieu de la prétention à l'existence de ces essences, et du même coup la condition de l'existentiabilité : l'existentiable est un possible qui existe en tant et en tant seulement qu'il est dans l'entendement de Dieu. Voilà qui répond à la deuxième des trois objections que J. Hostler formule en 1973 à l'encontre de 1' «omne possibile exigit existere » et qui consistait à demander : si les mondes possibles sont seulement possibles, comment peuvent-ils avoir une exigence actuelle ${ }^{64}$ Les possibles ont une réelle existence « in quadam ut sic dicam regione idearum, nempe in ipso Deo ${ }^{65}$, ce qui revient à interpréter le «omne possibile exigit existere» comme une description métaphorique du choix divin ${ }^{66}$.

La question se pose alors de savoir si l'on doit considérer l'existentiabilité comme un intermédiaire entre potentia et actus, entre le possible et l'existant. L'objection devrait être immédiate : l'existentiabilité ne peut en aucun cas constituer un intermédiaire entre la possibilité et l'existence, puisqu'elle n'est autre que la possibilité elle-même, et rien de plus. Il en va effectivement ainsi dans le référentiel leibnizien. On notera que les interprètes qui en font un intermédiaire, tel Heidegger qui écrit «La vis, eu égard à la traditionnelle distinction de potentia et actus, y est caractérisée pour ainsi dire en tant qu'une essence intermédiaire entre l'une et l'autre $»^{67}$, le font dans le référentiel aristotélicien de « la traditionnelle distinction de potentia et $a c t u{ }^{68}$. En somme, l'existentiabilité n'est pas un intermédiaire entre les notions leibniziennes de possibilité et d'existence, pour la bonne raison qu'elle est cette possibilité, mais peut être entendue comme un intermédiaire entre les notions aristotéliciennes de potentia et actus.

\section{L'existentiabilité est proportionnelle à la perfection}

Les possibles, on le sait, sont divers : « les choses sont possibles en beaucoup de manières » 69 aime à rappeler l'auteur. Que dire, dès lors, de leur prétention à l'existence ? Est-elle la même en chacun d'eux ? Tous les possibles tendent à l'existence: "prétendre que certaines essences possèdent cette inclination alors que d'autres ne la possèdent pas, c'est avancer quelque chose sans raison, puisqu'il semble qu'en général on rapporte l'existence à toutes les essences de la même

\footnotetext{
60 Sans-titre, sur Descartes ; GP IV 406, 1.

${ }^{61}$ M. Heidegger, 1971, t. II, p. 358.

62 ibid.

63 «Pour appeler quelque chose possible, ce m'est assez qu'on en puisse former une notion, quand elle ne serait que dans l'entendement divin, qui est pour ainsi dire le pays des réalités possibles » (Lettre à Arnauld, du $1^{\text {er }}$ juillet 1686 , GP II 55) ; «l'entendement de Dieu est la région des vérités éternelles » (Monadologie, §43).

64 J. Hostler, 1973, p. 282.

65 De Rerum originatione radicali, GP VII 305.

66 Voir J. Hostler, 1973, p. 284.

${ }^{67}$ M. Heidegger, 1971, t. II, p. 356.

68 C'est moins clair chez M. de Gaudemar, 1994, p. 48, qui parle d' «intermédiaire entre la possibilité et l'existence en acte », même si elle fait aussitôt référence à la dynamis aristotélicienne.

${ }^{69}$ Réfutation inédite de Spinoza, in Leibniz, 1999, p. 31.
} 
manière $»^{70}$; mais y tendent-ils également ? En droit, oui : «tous les possibles tendent d'un droit égal à exister $»^{71}$. Mais en fait, non : «chaque possible ayant droit de prétendre à l'existence à mesure de la perfection qu'il enveloppe $\gg^{72}$, leur tendance est proportionnelle à leur perfection respective. Le «à mesure » signifie effectivement «proportionnellement à », comme en témoigne la traduction latine de 1721, qui dira «pro ratione perfectionis quam involvit ». « qu'il enveloppe » signifie quant à lui, comme le note E. Boutroux, « qu'il contient à l'état d'enveloppement. Les possibles, avant le fiat divin, sont comparables à des germes, où est entièrement préformé l'être qui est susceptible d'en naître. L'existence n'est que le développement de ces germes $\gg^{73}$.

Chaque possible, donc, enveloppe, c'est-à-dire contient, une perfection qui lui est propre. Que dire de cette perfection? Qu'elle est chez Leibniz comme chez de nombreux autres rationalistes de son siècle la quantité d'essence ou de réalité : «la perfection n'est autre chose que la quantité d'essence $»^{74}$. Spinoza, après Descartes, écrit la même chose : «puisque pouvoir exister, c'est puissance, il s'ensuit que plus il y a de réalité dans la nature d'une chose, plus elle a par elle-même de forces pour exister " $^{75}$. La perfection, en somme, est le principe de l'existence : « de même que la possibilité est le principe de l'essence, de même la perfection ou le degré de l'essence (défini par le maximum de compossibles) est le principe de l'existence $\gg^{76}$.

Récapitulons : " tous les possibles, c'est-à-dire tout ce qui exprime une essence ou réalité possibles, tendent d'un droit égal à l'existence, en proportion de la quantité d'essence ou de réalité, c'est-à-dire du degré de perfection qu'ils impliquent. Car la perfection n'est autre chose que la quantité d'essence ${ }^{77}$.

\section{Le caractère mécanique de l'existentiabilité}

Dans plusieurs textes, Leibniz insiste sur l'aspect mécanique de la prétention des possibles à l'existence, en recourant à ce qu'il appelle lui-même une «comparaison d'un mécanisme métaphysique déterminant avec le mécanisme physique des corps graves ${ }^{78}$. En voici deux morceaux :

\footnotetext{
toute essence ou réalité exige l'existence comme tout effort exige le mouvement ou l'effet, si bien sûr rien ne l'empêche. (...) Ainsi, de même que sur une balance chaque poids s'efforce et tend sur son bras en fonction de sa pesanteur, et exige de descendre, si rien n'empêche, et que le plus pesant l'emporte, de même chaque chose aspire à l'existence en fonction de sa perfection, et la plus parfaite l'obtient. Par suite, tout possible existe, si un plus parfait n'en empêche l'existence. 79
}

Ainsi encore et surtout en mécanique ordinaire, de l'action de plusieurs graves concourant entre eux résulte le mouvement par lequel en fin de compte se réalise la plus grande descente. Et de même que tous les possibles tendent d'un droit égal à exister, en proportion de leur réalité, ainsi tous les poids tendent aussi d'un droit égal à descendre, en proportion de leur gravité ; de même qu'ici se produit le mouvement

\footnotetext{
70 Sur les vérités premières, in Leibniz, 1998, pp. 447-448.

${ }^{71}$ De Rerum originatione radicali, GP VII 304, in Leibniz, 2001b, pp. 177-179. Voir aussi GP VII 303, p. 173.

72 Monadologie, $\$ 54$.

${ }^{73}$ E. Boutroux, notes à Leibniz, 1880, p. 172, n. 3.

74 De rerum originatione radicali, GP VII 303, in Leibniz, 2001b, p. 173. Voir aussi « Perfectio est gradus < seu quantitas > realitatis » (Leibniz, 1948, p. 11).

75 Ethique, I, prop. 11, scolie, in Spinoza, 1954, p. 319. Voir également et avant lui Descartes, Méditation III, AT IX 32-33.

76 De Rerum originatione radicali, GP VII 304, in Leibniz, 2001b, p. 179.

77 De Rerum originatione radicali, GP VII 303, in Leibniz, 2001b, p. 173.

${ }^{78}$ De Rerum originatione radicali, GP VII 304, in Leibniz, 2001b, p. 179.

${ }^{79}$ Remarques générales, in Leibniz, 1998, p. 455.
} 
dans lequel se remarque le maximum de descente des graves, de même le monde qui se réalise est celui qui réalise le maximum de possibles. ${ }^{80}$

L'auteur lui-même introduit une objection pour l'écarter aussitôt et aisément : cette comparaison «pèche cependant en ceci que les corps graves pourvus de force existent véritablement, tandis que les possibilités ou essences, antérieurement à l'existence ou hors d'elle, sont imaginaires ou fictives, et que par suite on ne saurait en tirer aucune raison de l'existence ${ }^{81}$. Conformément à la nature existentiable des possibles en question, Leibniz répond en niant leur ficticité, puisqu'ils « existent, pour ainsi dire », dans l'entendement divin.

\title{
Les possibles tendent-ils réellement vers l'existence? Literalist view vs. Figurative view
}

Pour certains commentateurs, les possibles leibniziens ne tendent pas réellement vers l'existence, mais seulement métaphoriquement - stratagème que l'auteur utiliserait pour signifier plus abstraitement que l'essence tend vers l'existence. C'est ce que l'on nomme la Figurative view. Nicolas Grimaldi l'exprime ainsi :

\begin{abstract}
En effet, ce ne sont pas les pures essences ou les purs possibles qui tendent en soi à l'existence. C'est seulement la volonté de Dieu qui tend à faire exister chacun d'eux à proportion de la perfection qu'il renferme. Le résultat étant d'ailleurs le même, Leibniz peut bien métonymiquement attribuer à l'effet les caractères de la cause ; comme se passe finalement comme si «l'essence (tendait) par elle-même à l'existence». Leibniz prend soin cependant de préciser le caractère métaphorique de ce déplacement : « il y a, dans les choses possibles ou dans la possibilité même, c'est-à-dire dans l'essence, une certaine exigence d'existence, ou bien, pour ainsi dire, une prétention à l'existence... » (De rerum originatione radicali, trad. P. Schrecker, $\mathrm{p}$. 85). Cf. aussi Théodicée, $\$ 201$, G. vi.236 : « il y a un combat entre tous les possibles... Il est vrai que tout ce combat ne peut être qu'idéal, c'est-à-dire il ne peut être qu'un conflit de raisons dans l'entendement le plus parfait $\gg .82$
\end{abstract}

La première citation (de rerum) ne prouve rien : le «pour ainsi dire» de Leibniz ne s'applique pas à l'exigence, ou la prétention, elle-même, mais à l'usage du mot «prétention » pour désigner cette exigence. La seconde (Théodicée) ne porte pas sur l'exigence, mais sur la somme de la pluralité et de la rivalité de ces exigences, qui donc forment un combat ; et l'idéalité du combat ne signifie pas que l'exigence est métaphorique, mais seulement qu'elle n'est, elle aussi, qu'idéale.

La Figurative view semble ainsi faire deux confusions, qui ne sont pas dénuées d'intérêt car, malgré tout, la fausseté de sa conclusion est dérivée de deux vérités. Elle tire la non-réalité de la tendance des possibles vers l'existence (son caractère métaphorique), d'une part, du fait que les possibles ne tendent pas en soi et par eux-mêmes vers l'existence mais par Dieu, si l'on peut dire, ce qui est exact mais n'implique pas que ces possibles ne tendent pas réellement vers l'existence, à moins de confondre «tendre en soi » et «tendre réellement», c'est-à-dire inséité et réalité. D'autre part, elle tire sa conclusion du fait que cette tendance, du même coup, n'est qu'idéale, ce qui est aussi exact, mais qui n'implique pas davantage sa non réalité, à moins de confondre idéalité et nonréalité. Or, c'est précisément de cela que se défend Leibniz dans la réponse qu'il fait à Arnauld concernant la question de « la réalité des substances simplement possibles »: être idéal, c'est-à-dire être dans l'entendement de Dieu, n'est pas pour autant n'être qu'une chimère, comme nous l'avons

\footnotetext{
${ }^{80}$ De Rerum originatione radicali, GP VII 304, in Leibniz, 2001b, pp. 177-179.

${ }^{81}$ De Rerum originatione radicali, GP VII 304-305, in Leibniz, 2001b, p. 179.

82 N. Grimaldi, 1980, p. 182, n. 31.
} 
JB Jeangène Vilmer, « Possibilité et existentiabilité... », Revue philosophique de Louvain, 2006

vu précédemment ; ce n'est pas davantage n'être qu'une métaphore. On peut donc s'en tenir à une lecture littérale, dite Literalist view ${ }^{83}$.

\section{CONCLUSION.}

\section{De l'existentiabilité à l'existentialisme}

L'existentiabilité est le moyen terme d'un syllogisme implicite qui identifie d'une certaine manière l'essence à l'existence : (1) l'essence est identifiée à l'existentiabilité. L'existentiabilité est tellement essentielle à l'essence qu'elle l'est : « la nature de la possibilité ou de l'essence est de prétendre à l'existence $»^{84}$. (2) Or, l'existence est identifiée à l'existentiabilité. A propos de la proposition «que tout possible prétend exister », Leibniz note effectivement en marge : "Si l'existence était autre chose qu'une prétention de l'essence, alors elle aurait elle-même une essence et quelque chose de nouveau viendrait s'ajouter aux choses, à propos de quoi on pourrait à nouveau se demander si cette essence existe ou n'existe pas et pourquoi celle-ci plutôt que celle-là $»^{85}$. (3) Donc, d'une certaine manière, l'essence et l'existence sont identifiées l'une à l'autre. Et cette manière est le moyen terme qui s'efface dans le syllogisme : l'existentiabilité.

Ce résultat n'a rien d'exceptionnel : il est bien connu de la plupart des interprètes. Ainsi Jalabert interprète-t-il la tendance des possibles vers l'existence comme « une certaine identité de l'essence et de l'existence », totale en Dieu, partielle chez les créatures : "Le possible logique est un existant virtuel; il enveloppe une certaine exigence d'existence. On peut parler d'une certaine identité de l'essence et de l'existence. En Dieu, cette identité est absolue ; son essence est existence. Chez les êtres contingents l'essence est existence virtuelle et tendance à exister en acte. C'est la dynamique des essences qui est la source des existences. Si l'essence n'était pas existence à quelque titre, elle ne serait pas 'l'origine radicale des choses' ${ }^{86}$.

Il nous semble néanmoins important d'insister sur la conséquence que l'essence (la possibilité) n'est du même coup chez Leibniz absolument pas indépendante de l'existence : sa prétention à l'existence (son existentiabilité) lui est trop essentielle pour que l'on puisse l'en détacher ${ }^{87}$. C'est donc à juste titre que J.-L. Marion critique sur ce point la lecture de Cassirer, qui défend l'indépendance: «Cette césure peut surprendre d'abord parce que toute la théorie leibnizienne de la possibilité vise à rendre pensable le passage à l'effectivité en vertu de l' « exigence d'existence »; car c'est à partir de soi seule que «possibilitas exig[i]t existentiam ${ }^{88}$. En détachant à ce point l'essence de son exigence d'existence, Cassirer ne déforme-t-il pas la

\footnotetext{
83 C. Shields, 1994, p. 18, répond à Blumenfeld en lui montrant que sa critique de l'interprétation littérale est toute entière basée sur une prémisse (« The literalist interpretation implies necessitarianism »), qui assimile fallacieusement interprétation littérale et interprétation nécessitariste. Or, selon Shields, la Literalist view n'est pas forcément nécessitariste. Le nécessitarisme est la thèse selon laquelle chaque existence est nécessaire (voir L. Peña, 1997, p. 429 : «According to Leibniz existence, every existence, is necessary. That is why he defines (...) ens as 'possible' »), par opposition au contingentisme (voir par exemple Robinet et J. Skosnik, 1980).

${ }^{84}$ Note en marge de Sur les vérités premières, in Leibniz, 1998, pp. 447-448.

85 Sur les vérités premières, in Leibniz, 1998, p. 447.

86 J. Jalabert, 1968, p. 13, renvoie à «Deus est Ens, de cujus essentia est existentia », GP I 212, pour l'identité totale en Dieu et à De rerum originatione radicali, GP VII 302, pour l'identité partielle chez les créatures. Voir aussi, p. $14:$ : Il y a identité de l'être et de l'agir ; mais, dans l'être et l'agir, il y a des formes et des degrés. L'existence proprement dite, c'est la forme supérieure de l'agir, celui où l'exigence d'existence s'épanouit en acte plein ».

${ }^{87}$ L. Peña, 1997, p. 427 : «In summary, for Leibniz, essence grounds existence, but essence itself needs an existential support ».

${ }^{88}$ Marion cite «XXIV Propositiones », in Leibniz, 1903, p. 534, et renvoie en note à GP VII 195 et 303.
} 
doctrine de l'essence elle-même ? ${ }^{89}$. C'est effectivement déformer la théorie leibnizienne de la possibilité que de la concevoir indépendante de l'existence. Et nous allons plus loin : non seulement l'essence (la possibilité) est dépendante de l'existence, mais encore elle lui est d'une certaine manière (l'existentiabilité) identifiée.

Plus généralement, l'existentiabilité leibnizienne, c'est-à-dire la part d'existence dans l'essence, parce qu'elle est la manifestation d'une continuité entre l'essence et l'existence, appelle à refuser, avec J.-P. Paccioni et avant lui J.-F. Courtine ${ }^{90}$, le dualisme simpliste qui voudrait qu'on oppose, à la suite de L'être et l'essence d'E. Gilson, les «philosophies de l'essence » (essentialisme) aux «philosophies de l'existence » (existentialisme). L'existentiabilité est la preuve qu'il n'est pas d'essentialisme leibnizien sans existentialisme.

Au sein même des études leibniziennes, maintenant, il convient pour les mêmes raisons de rejeter le dualisme que certains interprètes établissent entre possibilisme et actualisme. F. Nef oppose le domaine possibiliste, dans lequel « les possibles ne sont classés que par leur prétention à l'existence», et le domaine actualiste, dans lequel «le possible n'est pensé ou décrit que relativement à de l'actuel », pour finalement conclure que «L'espace logique des possibles a donc une structure possibiliste AVANT la création et actualiste APRES ${ }^{91}$. L'objection est simple : à moins de présupposer que «l'existence » évoquée dans le domaine possibiliste n'est pas la même chose que «l'actualité » évoquée dans le domaine actualiste, dans les deux cas le possible n'est pensé que relativement à l'existence-actualité. Le soi-disant possibilisme, tel qu'il est décrit ici, est donc absorbé par l'actualisme. On peut même aller plus loin, et mettre au défi l'auteur de défendre un «possibilisme» pur, pur comme la coupure qu'il évoque entre un AVANT la création et un APRES. Car à aucun moment le possible n'est pensé indépendamment de l'actuel - c'est-à-dire indépendamment de l'existence : "Je trouve aussi fort solide ce que vous dites ensuite, qu'on ne conçoit jamais aucune substance purement possible que sous l'idée de quelqu'une (ou par les idées comprises dans quelqu'une) de celles que Dieu a créées ${ }^{92}$. C'est la raison pour laquelle la possibilité peut se démontrer a posteriori par l'expérience, c'est-à-dire par l'actualité : «Tout ce qui est actuel, peut être conçu comme possible »; «ce qui existe actuellement ne saurait manquer d'être possible ${ }^{93}$. Ainsi le possible est-il appréhendé par rapport à l'actuel. Avant la création, son rapport à l'actuel est virtuel, c'est l'existentiabilité (existence en puissance). Après la création, son rapport à l'actuel est actuel, c'est l'existence (existence en acte). Mais dans les deux cas, donc toujours, il a un rapport à l'actuel. Autrement dit : il n'y a chez Leibniz que de l'actualisme ${ }^{94}$.

\footnotetext{
89 J.-L. Marion, 1990, p. 40.

90 Voir notamment J.-P. Paccioni, 2002, p. 65, et J.-F. Courtine, 1990, p. 9.

${ }^{91}$ F. Nef, 1999, pp. 292-293.

92 Lettre à Arnauld du 14 juillet 1686, GP II 55.

93 Respectivement Lettre à Arnauld du 14 juillet 1686, GP II 55 et Lettre à Thomas Burnett de Kemney, 1699, GP III 257 (ocpn). Notons que la possibilité peut également se démontrer a priori. Le texte complet de cette dernière citation est : « la marque d'une idée vraie et réelle est lorsqu'on en peut démontrer la possibilité, soit a priori en donnant ses réquisits, soit a posteriori par l'expérience : car ce qui existe actuellement ne saurait manquer d'être possible ». Voir aussi « Je tiens que la marque d'une idée véritable est qu'on puisse prouver la possibilité, soit a priori en concevant sa cause ou sa raison, soit a posteriori, lorsque l'expérience fait connaître qu'elle se trouve effectivement dans la nature » (GP II 63). La démonstration a priori de la possibilité de l'idée vraie et réelle ne constitue en rien une objection à l'actualisme car l'on ne peut jamais partir que d'une idée «vraie et réelle », donc en un sens actuelle, pour chercher « sa cause ou sa raison ». L'actuel précède toujours chronologiquement le possible : il en est la condition d'accès. Voir aussi GP IV 403, GP IV 425 et GP V 272.

94 S. Madouas, 1999, p. 386 : «Leibniz est actualiste et non pas possibiliste ».
} 
JB Jeangène Vilmer, « Possibilité et existentiabilité... », Revue philosophique de Louvain, 2006

\section{BIBLIOGRAPHIE}

\section{1. Éditions des æuvres de Leibniz}

A : Leibniz, Sämtliche Schriften und Briefe herausgegeben von der Akademie der Wissenschaften der DDR ; Sechste Reihe (Philosophische Schriften) :

- Erster Band (1663-1672), herausgegeben von der Preussischen Akademie der Wissenschaften, Berlin : Otto Reichl Verlag Darmstadt, 1930 [noté A VI-1, suivi du numéro de la page].

- Zweister Band (1663-1672), herausgegeben von der Leibniz-Forschungsstelle der Universität Münster, Berlin : Akademie Verlag, 1966, [noté A VI-2, suivi du numéro de la page].

- Vierter Band (1677 - Juni 1690), herausgegeben von der Leibniz-Forschungsstelle der Universität Münster, Berlin : Akademie Verlag, 1999, Teil A [noté A VI-4-A, suivi du numéro de la page] ; Teil B [noté A VI-4-B, suivi du numéro de la page] ; Teil C [noté A VI-4-C, suivi du numéro de la page] ; (le Teil D est un index).

GP : Leibniz, Die philosophischen Schriften, herausgegeben von C. I. Gerhardt, Berlin : Weidmannsche Buchhandlung, 1875-1890, 7 vol., réed. Hildesheim : G. Olms, 1978.

GM : Leibniz, Matematische Schriften, herausgegeben von C. I. Gerhardt, réed. Hildesheim : G. Olms, 1962.

LEIBNIZ (1880). Monadologie, Paris, Delagrave.

LEIBNIZ (1903). Opuscules et fragments inédits de Leibniz, extraits des manuscrits de la Bibliothèque royale de Hanovre, Louis Couturat (ed.), Paris, Alcan.

LEIBNIZ (1940). Euvres choisies, L. Prenant (ed.), Paris, Garnier.

LEIBNIZ (1948). Textes inédits d'après les manuscrits de la Bibliothèque provinciale de Hanovre, Gaston Grua (ed.), Paris, PUF.

LEIBNIZ (1970). Confessio Philosophi. La profession de foi du philosophe, Y. Belaval (ed.), Paris, Vrin.

LEIBNIZ (1995). L'estime des apparences, M. Parmentier (ed.), Paris, Vrin.

LEIBNIZ (1996). Principes de la Nature et de la Grâce, Monadologie, et autres textes 1703-1716, C. Frémont (ed.), Paris, Flammarion.

LEIBNIZ (1998). Recherches générales sur l'analyse des notions et des vérités. 24 thèses métaphysiques et autres textes logiques et métaphysiques, J.-B. Rauzy (ed.), Paris, PUF.

LEIBNIZ (1999). Réfutation inédite de Spinoza. Remarques critiques de Leibniz sur un livre de J. G. Wachter à propos de la philosophie cachée des hébreux, d'après le manuscrit original de la Bibliothèque royale de Hanovre, M. de Gaudemar (ed.), Paris, Actes Sud.

LEIBNIZ (2001a). Discours de métaphysique et autres textes 1663-1689, C. Frémont (ed.), Paris, Flammarion.

LEIBNIZ (2001b). Opuscules philosophiques choisis, P. Schrecker (ed.), Paris, Vrin.

\section{Littérature secondaire}

COURTINE J.-F. [1990], Suarez et le système de la métaphysique, Paris, PUF.

COUTURAT L. (1902), «Sur la métaphysique de Leibniz », Revue de métaphysique et de morale, 10, p. 1-25.

D’AGostino F. B. (1994), « Leibniz on Compossibility and Relational Predicates », The Philosophical Quartely, 26:103, 1976 ; in Gottfried Wilhelm Leibniz. Critical Assessments, vol. II, éd. par R. S. Woolhouse, London-NY, Routledge.

GAUDEMAR M. de (1994), De la puissance au sujet, Paris, Vrin.

GRIMALDI N. (1980), «Rationalité et temporalité chez Leibniz », Revue de métaphysique et de morale, 85:2, p. 178-192.

GUÉROULT M. (1947), «La constitution de la substance chez Leibniz », Revue de métaphysique et de morale, $52: 1$, p. $55-78$.

HACKING I. (1971), «The Leibniz-Carnap Program for Inductive Logic », The Journal of Philosophy, 48:19, october 7, p. 597-610.

- (1975). The Emergence of Probability, Cambridge, Cambridge University Press.

HEIDEGGER M. (1971). Nietzsche, Günther Neske Verlag, 1961, t. II, VIII, traduit par P. Klossowski, Paris, Gallimard.

HOSTLER J. (1973), « Some remarks on 'omne possibile exigit existere' », Studia Leibnitiana, vol. 5, pp. $281-285$.

JAGODINSKI (1913). Leibnitiana. Elementa philosophiae arcanae, de summa rerum, Kazan. 
JB Jeangène Vilmer, « Possibilité et existentiabilité... », Revue philosophique de Louvain, 2006

JALABERT J. (1968), «Les Notions d'Essence et d'Existence dans la Philosophie de Leibniz », Akten des Internationalen Leibniz-Kongresses, Hannover, 14-19 November 1966, Band I, Metaphysik - Monadenlehre, Studia Leibnitiana Supplementa, vol. I, Wiesbaden : Steiner, pp. 13-21.

KALINOWSKI G. (1983), «Leibniz et les sémantiques des mondes possibles », in (coll.), Leibniz, Werk und Wirkung. IV. Internationaler Leibniz-Kongreß, Hannover, 14 bis 19 November 1983, pp. 336-343.

KNECHT H. H. (1981). La logique de Leibniz. Essai sur le rationalisme baroque, Lausanne, L'Age d'Homme.

KRÜGER L. (1981), «Probability in Leibniz. On the internal coherence of a dual concept », Archiv für Geschichte der Philosophie, 63, pp. 47-60.

MADOUAS S. (1999), "L'Adam vague et la constitution des mondes possibles: une pensée modale de l'individu », in L'actualité de Leibniz : les deux labyrinthes, éd. par D. Berlioz et F. Nef, Décade de Cerisy La Salle, 15-22 juin 1995, Studia Leibnitiana supplementa, vol. 34, Stuttgart, Steiner.

MARION J.-L. (1990), «L'interprétation criticiste de Descartes et Leibniz: (Critique d'une critique) », in Ernst Cassirer : De Marbourg à New York. L'itinéraire philosophique, éd. par J. Seidengart, Actes du colloque de Nanterre, 12-14 octobre 1988, Paris, Cerf, pp. 29-42.

MOREAU J. (1956), L'univers leibnizien, Paris, Vitte.

NEF F. (1999), «La philosophie modale de Leibniz est-elle cohérente ? Essai sur des problèmes d'interprétation de notions modales leibniziennes à propos du mythe de Sextus et de l'oracle de Kegila », in L'actualité de Leibniz : les deux labyrinthes, éd. par D. Berlioz et F. Nef, Décade de Cerisy La Salle, 15-22 juin 1995, Studia Leibnitiana supplementa, vol. 34, Stuttgart, Steiner, pp. 277-305.

PACCIONI J.-P. (2002), «L'aptitude à exister et la métaphysique wolffienne », Archives de philosophie, 65, pp. 6580.

PENA L. (1997), «Essence and Existence in Leibniz’s Ontology », Synthesis Philosophica, 12:2, pp. 415-431.

PUTNAM H. (1973), « Meaning and Reference », The Journal of Philosophy, 70:18, pp. 699-711.

RONCAGLIA G. (1988), « Modality in Leibniz's Essays on Logical Calculus of April 1679 », Studia Leibnitiana, 20:1, pp. 43-62.

SARTRE J.-P. (1943). L'être et le néant, Paris, Gallimard.

SHIELDS C. (1994), «Leibniz's Doctrine of the Striving Possibles », Journal of the History of Philosophy, 24:3, 1986, p. 343-357 ; in Gottfried Wilhelm Leibniz. Critical Assessments, vol. II, éd. par R. S. Woolhouse, London-NY : Routledge, pp. 14-28.

SKOSNIK J. (1980), «Leibniz and Russell on Existence and Quantification Theory», Canadian Journal of Philosophy, 10:4, pp. 681-720.

SPINOZA (1954). Euvres complètes, R. Caillois, éd. par M. Francès et R. Misrahi, Paris, Gallimard.

VAN FRAASSEN B. (1977), « Relative Frequencies », Synthese, 34:2, pp. 133-166.

WILSON M. (1971), « Possibility, Propensity, and Change : some doubts about the Hacking Thesis », The Journal of Philosophy, 48:19, october 7, pp. 610-617.

WITTGENSTEIN Ludwig (1961). Tractatus Logico-Philosophicus, Paris, Gallimard. 